\title{
Vitamin-D deficiency is associated with chronic bacterial colonisation and disease severity in bronchiectasis
}

\author{
James D Chalmers, ${ }^{1}$ Brian J McHugh, ${ }^{1}$ Catherine Docherty, ${ }^{2}$ John R W Govan, ${ }^{2}$ \\ Adam T Hill ${ }^{1,3}$
}

\begin{abstract}
- Additional supplementary tables are published online only. To view these files please visit the journal online (http:/l dx.doi.org/10.1136/thoraxjnl2012-202125)

${ }^{1}$ The University of Edinburgh/ MRC Centre for Research Inflammation, The Queen's Medical Research Institute, Edinburgh, UK

${ }^{2}$ Cystic Fibrosis Group, University of Edinburgh, Schoo of Medicine and Veterinary Medicine, Edinburgh, UK ${ }^{3}$ Department of Respiratory Medicine, Royal Infirmary of Edinburgh, Edinburgh, UK
\end{abstract}

\section{Correspondence to} Dr James D Chalmers, The University of Edinburgh/MRC Centre for Research Inflammation, The Queen's Medical Research Institute, 47 Little France Crescent, Edinburgh EH16 4TJ, UK; jamesdchalmers@googlemail. com

Received 7 May 2012 Revised 8 September 2012 Accepted 14 September 2012 Published Online First 16 October 2012

\footnotetext{
To cite: Chalmers JD, McHugh BJ, Docherty C, et al. Thorax 2013, 68, 39-47.
}

\begin{abstract}
Introduction Vitamin-D deficiency has been linked to an increased risk of respiratory infections. The objective of this study was to determine the frequency and clinical importance of vitamin-D deficiency in patients with bronchiectasis.

Methods 25-hydroxyvitamin-D was measured by immunoassay in 402 stable patients with bronchiectasis. Patients were classified as vitamin-D deficient (serum 25-hydroxyvitamin-D $<25 \mathrm{nmol} / \mathrm{l}$ ), insufficient (25 nmol/ $1-74 \mathrm{nmol} / \mathrm{l})$ or sufficient $(\geq 75 \mathrm{nmol} / \mathrm{l})$. Disease severity was assessed, including exacerbation frequency, measurement of airway inflammatory markers, sputum bacteriology and lung function over 3 years follow-up.

Results $50 \%$ of bronchiectasis patients were vitamin-D deficient, $43 \%$ insufficient and only $7 \%$ sufficient. This compared to only $12 \%$ of age and sex matched controls with vitamin-D deficiency $(p<0.0001)$. Vitamin-D deficient patients were more frequently chronically colonised with bacteria $(p<0.0001), 21.4 \%$ of vitamin-D deficient subjects were colonised with Pseudomonas aeruginosa compared to $10.4 \%$ of insufficient patients and $3.6 \%$ of sufficient patients, $p=0.003$. Vitamin-D deficient patients had lower $\mathrm{FEV}_{1} \%$ predicted

\section{Key messages}

What is the key question

- Vitamin-D deficiency has been linked to an increased risk of respiratory infections. The key objective of this study was to determine the frequency and clinical importance of vitamin-D deficiency in patients with bronchiectasis.

\section{What is the bottom line}

- Vitamin-D deficiency is common in bronchiectasis and is associated with a higher frequency of bacterial colonisation, more exacerbations and worse quality of life.

\section{Why read on}

- We have explored the effects of Vitamin-D deficiency on neutrophil phagocytosis, sputum concentrations of LL-37 and Vitamin-D Binding Protein. We propose that Vitamin-D be evaluated as a potential therapy in bronchiectasis.
\end{abstract} $(p=0.002)$, and more frequent pulmonary exacerbations $(p=0.04)$. Vitamin-D deficient patients had higher sputum levels of inflammatory markers and demonstrated a more rapid decline in lung function over 3 years follow-up. Defects in neutrophil function and assessment of airway LL-37 levels did not provide a mechanistic explanation for these findings. Vitamin-D deficient patients had, however, higher levels of VitaminD Binding Protein in sputum sol.

Conclusions Vitamin-D deficiency is common in bronchiectasis and correlates with markers of disease severity. The mechanism of this association is unclear.

\section{BACKGROUND}

Bronchiectasis is a chronic, debilitating, lung disease characterised by a vicious cycle of airway inflammation and bacterial colonisation. ${ }^{1}$ There are no effective long term treatments apart from antibiotics and chest clearance techniques. ${ }^{2}$ The underlying cause of bronchiectasis is unknown in the majority of patients. ${ }^{3}$

Chronic colonisation with bacterial pathogens such as Haemophilus influenzae and Pseudomonas aeruginosa leads to progressive lung damage, recurrent exacerbations and premature death. ${ }^{4-6}$
Understanding why some patients develop chronic bacterial colonisation and other patients experience a more benign course of bronchiectasis is the key to developing new treatments.

It is estimated that one billion people worldwide are deficient in vitamin-D and in the UK, a nationwide survey demonstrated that more than $50 \%$ of the adult population had vitamin-D insufficiency and $16 \%$ had severe deficiency during winter and spring. ${ }^{8}$ The highest rates of vitamin-D deficiency were observed in Scotland. ${ }^{7} 8$

Vitamin-D has potent effects on innate immunity in vitro with the vitamin-D receptor expressed on the majority of immune cells (monocytes, T-cells, B-cells and dendritic cells). Vitamin-D has been shown to reduce the production of pro-inflammatory cytokines ${ }^{9}$ and regulates the secretion of antimicrobial peptides such as cathelicidin (LL-37) which has potent antimicrobial activity against $P$ aeruginosa. ${ }^{10-12}$ Wang et $a l^{10}$ showed that vitamin-D treatment of inflammatory cells in vitro enhanced killing of Paeruginosa.

A mouse model of vitamin-D deficiency has recently shown that deficiency directly causes alterations in lung structure and reduced lung function. ${ }^{13}$ 
These effects of vitamin-D deficiency make it a relevant candidate to modify disease severity in bronchiectasis. Vitamin-D deficiency resulting from reduced sunlight exposure or poor diet is well recognised. ${ }^{14}$ It is also recognised however, alterations in vitamin-D binding protein (VDBP) may influence circulating vitamin-D levels. The level of VDBP is linked to circulating vitamin-D through alterations in vitamin-D bioavailability but also through single nucleotide polymorphisms in the VDBP gene that lead to both increased VDBP and reduced serum 25-hydroxyvitamin-D (25-OHD). ${ }^{15}$ Of direct relevance to bronchiectasis, high levels of airway VDBP has been shown to be pro-inflammatory, inducing macrophage activation and causing impaired neutrophil chemotaxis. ${ }^{16} 17$

The aim of this study was to determine if vitamin-D deficiency is associated with chronic bacterial colonisation, airway inflammation and disease severity in patients with bronchiectasis.

\section{METHODS}

Consecutive adult patients with non-cystic fibrosis bronchiectasis were recruited from a regional specialist clinic at the Royal Infirmary of Edinburgh, UK. The study was approved by the South East Scotland Research Ethics committee and all participants gave written informed consent.

Included patients had clinically significant bronchiectasis confirmed by high resolution CT scan. ${ }^{2}$ Exclusion criteria were age $<18$ years, poorly controlled asthma; active allergic bronchopulmonary aspergillosis, current smoking (within 2 years), cystic fibrosis, active mycobacterial disease, long term use of oral or nebulised antibiotic therapy or long term oral corticosteroid therapy at any dose. Full medication histories, including over the counter medication use, were obtained at study entry. No patients were included if they reported taking prescribed or over the counter calcium or vitamin-D supplements.

\section{Study design}

All patients were clinically stable and free from antibiotic therapy for at least 4 weeks at enrolment. Two hundred age and sex matched control subjects were recruited from the spouses and partners of patients attending the respiratory outpatient clinics at the Royal Infirmary of Edinburgh. Control subjects were not prescribed vitamin-D supplements either at any time prior to the study or during the study period.

To reduce the impact of seasonal variation in vitamin-D on measurements and clinical parameters all vitamin-D measurements were made on serum samples collected between October and March at the commencement of the study and clinical parameters were recorded annually.

\section{Measurement of serum vitamin-D}

Serum total (25-OHD-the main circulating form of vitamin-D) was measured using the enzyme immunoassay method (Immunodiagnostic systems, Boldon,UK) according to the manufacturer's instructions. Further details of the vitamin-D assay are provided in the online supplementary material. This assay correlates well with alternative methods such as radioimmunoassay and mass spectrometry, ${ }^{18}{ }^{19}$ has been used in clinical studies and has been validated using the Vitamin-D External Quality Assessment Scheme methodology. ${ }^{19}$

The definition of vitamin-D deficiency is the subject of debate. For the main analysis, a priori, vitamin-D deficiency was defined according to the kit manufacturer's recommendations and cut-offs previously used in clinical studies as $<25 \mathrm{nmol} / \mathrm{l}$ (equivalent to $<10 \mathrm{ng} / \mathrm{ml}$ ) with insufficiency defined as serum
25 -OHD. $25 \mathrm{nmol} / 1-74 \mathrm{nmol} / 1$ and $\geq 75 \mathrm{nmol} / 1$ was regarded as sufficient serum 25-OHD. A sensitivity analysis using a second definition of vitamin-D deficiency is presented in the online supplementary material. ${ }^{14}$ Measurements of baseline 25-OHD were performed in batch at the end of the study and therefore knowledge of patients 25-OHD status did not influence the patient's clinical management or assessments during follow-up.

\section{Bacteriology}

Spontaneous early morning sputum samples containing less than 10 squamous cells and more than 25 leukocytes per low power microscope field were acceptable. ${ }^{20}$ Sputum was separated from saliva and the sample split for bacteriology and assessment of airway inflammation markers. $1 \mathrm{ml}$ sputum was homogenised by treatment with dithiotreitol (Sputolysin; Calbiochem, San Diego, California, USA). Samples were processed for quantitative and qualitative bacteriology as described. ${ }^{21}$ Chronic colonisation was defined by the isolation of the same bacterial species in sputum on two or more occasions, at least 3 months apart in a 1 year period while clinically stable.

\section{Clinical assessments}

All stable patients had 6 monthly assessments and underwent spirometry (forced expiratory volume in $1 \mathrm{~s}\left(\mathrm{FEV}_{1}\right)$ forced vital capacity with the highest of three technically satisfactory measurements recorded) ${ }^{22}$ and patients completed the St. Georges Respiratory Questionnaire (SGRQ) ${ }^{23}$ and the Leicester cough questionnaire (LCQ). ${ }^{24}$ The LCQ has a score from 3 to 21 (a score of 3 is most severe cough) and the SGRQ has a score from 0 to 100 (100 indicates maximum impairment). The minimal important clinical difference is 1.3 Units and four Units respectively for these questionnaires. Severity of bronchiectasis was assessed by scoring high resolution computed tomography scans using a modified Reiff score. ${ }^{3}$ This takes into account the number of lobes involved (with the lingula scored as a separate lobe) and the severity of dilatation (tubular-1 point, varicose- 2 points, cystic-3 points). The maximum score is 18 . Exacerbations were quantified from patient histories and verified against general practitioner antibiotic prescription records. Exacerbations were defined as in the British Thoracic Society bronchiectasis guidelines as an acute deterioration with worsening of cough, increased sputum production or change in viscosity, increased sputum purulence with or without increasing wheeze, breathlessness or haemoptysis. ${ }^{2}$ Hospitalisation was recommended for patients meeting the British Thoracic Society criteria for inpatient management of exacerbations (development of cyanosis or confusion, breathlessness with respiratory rate $\geq 25 / \mathrm{min}$, circulatory failure, respiratory failure, temperature $\geq 38^{\circ} \mathrm{C}$, inability to take oral therapy, inability to cope at home and failure of oral antibiotic therapy). ${ }^{2}$

\section{Airway inflammation}

Unprocessed sputum, not treated with dithiotreitol, was ultracentrifuged at $50000 \times \mathrm{g}$ for $90 \mathrm{~min}$ at $4^{\circ} \mathrm{C}$. The sol phase was removed and immediately frozen at $-70^{\circ} \mathrm{C}$. Myeloperoxidase and neutrophil elastase activity were measured as described. ${ }^{21}$ Interleukin (IL)-8, tumour necrosis factor (TNF)- $\alpha$ and IL-1 $\beta$ were measured by commercially available ELISA $(\mathrm{R}+\mathrm{D}$ systems, Abingdon, UK) using kits that had been validated for sputum use according to established methodology. ${ }^{25}$ Sputum levels of LL-37 were measured using a commercial ELISA kit (Hycult Biotechnology,Uden, Netherlands). VDBP was measured in sputum using a commercial ELISA kit $(\mathrm{R}+\mathrm{D}$ systems, Abingdon, UK). 


\section{Follow-up}

Patients were followed up for 3 years to assess frequency of exacerbations (averaged per year), colonisation status, decline in $\mathrm{FEV}_{1}$ and survival status.

\section{Neutrophil phagocytosis assays}

Peripheral blood neutrophils were isolated from patients with bronchiectasis by percoll-gradient density centrifugation as described. ${ }^{26}$ A flow cytometry based phagocytosis assay was used. ${ }^{27}$ FITC labelled $P$ aeruginosa and Eschericia coli were opsonised with $10 \%$ pooled serum from healthy donors at $37^{\circ} \mathrm{C}$ for $1 \mathrm{~h}$. Opsonised bacteria were then added at a multiplicity of infection $10: 1$ to patient neutrophils $\left(0.5 \times 10^{6}\right.$ per experiment $)$. Phagocytosis was permitted at $37^{\circ} \mathrm{C}$ for $30 \mathrm{~min}$ and then terminated by placing the samples on ice. Excess bacteria were removed by washing in phosphate buffered saline and cells subsequently analysed by fluorescence activated cell sorting (FACS). To differentiate phagocytosed (intracellular) bacteria from adherent (extracellular bacteria), cells were incubated with $0.1 \%$ trypan blue to quench extracellular fluorescence. All analyses were performed on a FACSCalibur flow cytometer (BD Biosciences, San Jose, California, USA).

\section{Statistical analysis}

For comparisons of more than two groups of continuous data, one way ANOVA was used for comparison of normally distributed data with the Kruskal-Wallis test used for non-normally distributed data. Dunn's multiple comparison test was used to correct for multiple comparisons. Distribution was assessed by the D'Agostino and Pearson omnibus normality test. $\chi^{2}$ test was used for more than two groups of categorical data. To adjust for confounders of the relationship between vitamin-D status and chronic colonisation multivariable logistic regression analysis was used. Variables with a $\mathrm{p}$ value $<0.1$ on univariate analysis were entered into a logistic regression model using a forward stepwise approach. Final model fit was assessed using the Hosner-Lemeshow goodness of fit test. To investigate the relationship between vitamin D status and survival, Cox's proportional hazard model was used to estimate survivor functions with survival over 3 years follow-up as the dependent variable. Variables associated with mortality $(p<0.1)$ in univariate analysis were adjusted for in the model. To account for the potential impact of season on vitamin-D levels, the timing of blood sampling (October-March) was included in both multivariable models. For all analyses, $\mathrm{p}<0.05$ was considered statistically significant.

\section{RESULTS}

\section{Study population}

After exclusion of 82 patients currently receiving vitamin-D supplementation, the study included 402 patients with bronchiectasis. The baseline characteristics of the study population are shown in table 1 . Vitamin-D deficient patients were more frequently prescribed inhaled corticosteroids and were more frequently female. The online supplement shows the baseline characteristics using different Vitamin D cut-offs (see online supplementary table E1 $<50 \mathrm{nmol} / \mathrm{l}, \quad 51-69 \mathrm{nmol} / \mathrm{l}$ and $\geq 70 \mathrm{nmol} / \mathrm{l})$.

\section{Vitamin-D deficiency in patients and controls}

The median serum 25-OHD was $24.7 \mathrm{nmol} / \mathrm{l}$ in patients (IQR 16.7-45.4) and $45.3 \mathrm{nmol} / 1$ in control subjects (IQR 30.968.9), $\mathrm{p}<0.001$. Vitamin-D deficiency was very common in bronchiectasis patients. Fifty per cent (201) of patients were deficient according to the study definitions $(<25 \mathrm{nmol} / \mathrm{l}) \mathrm{com}-$ pared to $12 \%$ of controls. Forty-three per cent of bronchiectasis patients met the criteria for insufficiency $(25-75 \mathrm{nmol} / \mathrm{l})$ meaning that only $7 \%$ (28) of the bronchiectasis patients enrolled in the study had sufficient 25-OHD levels. Low 25-OHD levels were significantly more frequent in bronchiectasis patients compared to controls $(p<0.001)$. There were no significant differences in age (median 67 years IQR 58-73 vs median 65 years IQR $53-76, \mathrm{p}=0.2)$, gender $(41.3 \%$ male vs $45.0 \%, \mathrm{p}=0.5)$, comorbidities or the month of sampling $(\mathrm{p}=0.8)$ between the groups to explain these differences (see the online supplement table E2 and E3).

Table 1 Baseline characteristics of the study population

\begin{tabular}{|c|c|c|c|c|c|}
\hline Baseline characteristics & Study population & Deficient $<25 \mathrm{nmol} / \mathrm{l}$ & Insufficient $25 \mathrm{nmol} / /-74 \mathrm{nmol} / \mathrm{l}$ & Sufficient $\geq 75 \mathrm{nmol} / \mathrm{l}$ & $\mathrm{p}$ Value \\
\hline N & 402 & 201 & 173 & 28 & \\
\hline Age & $67(58-73)$ & $67(58-74)$ & $67(60-73)$ & $65(58-74)$ & 0.9 \\
\hline Gender ( $\%$ male) & $166(41 \%)$ & $71(35 \%)$ & $81(47 \%)$ & $14(50 \%)$ & 0.03 \\
\hline BMI $\mathrm{kg} / \mathrm{m}^{2}$ & $25.7(22.1-29.5)$ & $25.7(22.3-28.7)$ & $25.4(21.8-29.5)$ & $27.2(22.2-32.6)$ & 0.4 \\
\hline Ethnicity (\% Caucasian) & $391(97.3 \%)$ & $193(96.0 \%)$ & $170(98.3 \%)$ & $28(100 \%)$ & 0.3 \\
\hline \multicolumn{6}{|l|}{ Comorbidities } \\
\hline Chronic cardiac disease & $79(20 \%)$ & $44(22 \%)$ & $32(19 \%)$ & $3(11 \%)$ & 0.3 \\
\hline Chronic renal impairment & $20(5 \%)$ & $14(7 \%)$ & $5(3 \%)$ & $1(4 \%)$ & 0.2 \\
\hline Diabetes mellitus & $52(13 \%)$ & $29(14 \%)$ & $20(12 \%)$ & $3(11 \%)$ & 0.7 \\
\hline \multicolumn{6}{|l|}{ Causes of bronchiectasis } \\
\hline Idiopathic/post infective & $251(62 \%)$ & $123(61 \%)$ & $112(65 \%)$ & $16(57 \%)$ & 0.7 \\
\hline Previous TB & $70(17 \%)$ & $36(18 \%)$ & $31(18 \%)$ & $3(11 \%)$ & 0.6 \\
\hline Previous ABPA & $35(9 \%)$ & $17(9 \%)$ & $13(8 \%)$ & $5(18 \%)$ & 0.2 \\
\hline Connective tissue disease & $27(7 \%)$ & $14(7 \%)$ & $9(5 \%)$ & $4(14 \%)$ & 0.2 \\
\hline Inflammatory bowel disease & $12(3 \%)$ & $7(4 \%)$ & $5(3 \%)$ & $0(0 \%)$ & 0.6 \\
\hline Others & $7(2 \%)$ & $4(2 \%)$ & $3(2 \%)$ & $0(0 \%)$ & 0.8 \\
\hline \multicolumn{6}{|l|}{ Treatment } \\
\hline Inhaled corticosteroid use & $166(41 \%)$ & $101(50 \%)$ & $61(35 \%)$ & $4(14 \%)$ & $<0.001$ \\
\hline
\end{tabular}




\section{Vitamin-D deficiency correlates with chronic bacterial} colonisation of the airways

Figure 1, shows the frequency of chronic bacterial colonisation in bronchiectasis patients according to their 25-OHD status. The most frequently isolated organism during the study was Haemophilus influenzae. At baseline, 69.2\% of the study population met the criteria for chronic colonisation. Chronic colonisation with any bacterial species was more common in patients with vitamin-D deficiency than in patients with vitamin-D insufficiency or patients with sufficient vitamin-D status, $\mathrm{p}<0.0001$ (figure 1). The difference between vitamin-D deficient and
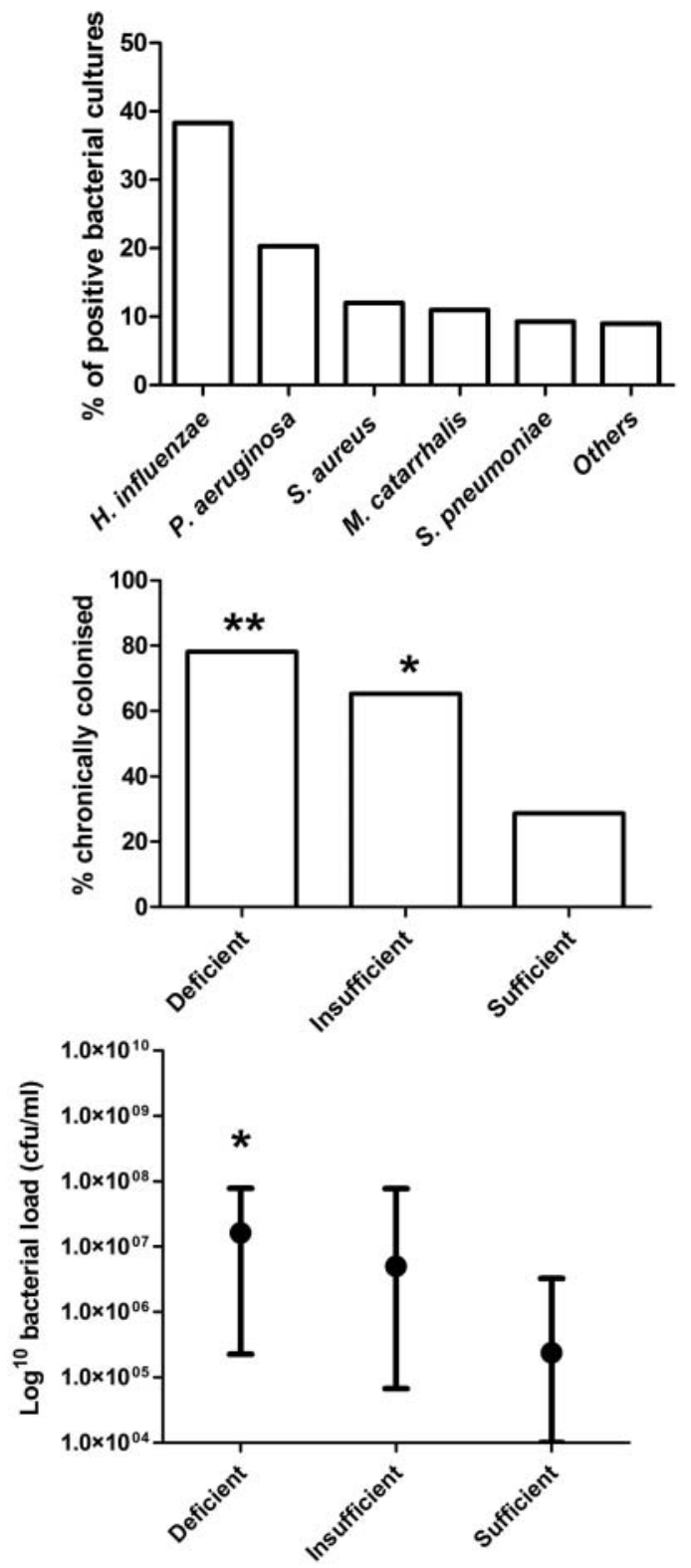

Figure 1 Association between vitamin-D status, chronic colonisation and bacterial load. (A) the frequency of bacterial isolates from 402 patients with bronchiectasis. (B) demonstrates a higher frequency of chronic bacterial colonisation in vitamin-D deficient patients compared to insufficient $(p=0.006)$ or sufficient patients $(p<0.0001)$. (C)

Quantitative bacteriology demonstrates a higher median bacterial load in sputum from vitamin-D deficient patients compared to insufficient and sufficient groups $(p=0.005)$. ${ }^{* *}$ indicates $p<0.05$ when compared to insufficient and sufficient groups. *indicates $p<0.05$ when compared to sufficient group. insufficient patients was also statistically significant, $\mathrm{p}=0.006$ by $\chi^{2}$ test. In logistic regression analysis vitamin-D deficiency was independently associated with chronic colonisation-adjusted OR $1.67,95 \%$ CI 1.10 to $2.56, p=0.01$. This analysis was adjusted for $\mathrm{FEV}_{1} \%$ predicted, radiological severity of bronchiectasis, gender, inhaled corticosteroid use and month of vitamin-D testing.

Quantitative bacteriology demonstrated a higher mean bacterial load in vitamin-D deficient patients compared to sufficient or insufficient patients $(\mathrm{p}<0.001)$.

Of the vitamin-D deficient subjects $21.4 \%$ were colonised with $P$ aeruginosa compared to $10.4 \%$ of insufficient patients and $3.6 \%$ of sufficient patients, $\mathrm{p}=0.003$.

Vitamin-D deficiency is associated with symptoms, exacerbations and quality of life

This secondary care population with bronchiectasis had significant impairment of quality of life and significant cough symptoms, with median (IQR) SGRQ and LCQ scores of 45.8 (26.9-62.7) and $13.9(10.4-17.4)$ respectively. There was a significant relationship between serum $25-\mathrm{OHD}$ and both SGRQ and LCQ with the differences between groups being significantly greater than the minimal clinically important difference (figure 2).

The median number of exacerbations was 3 per year (IQR 1-5). Vitamin-D deficient patients were more likely to suffer frequent outpatient exacerbations (median 3, IQR 2-5 for vitamin-D deficient patients compared to median 2, IQR 1-5 for insufficient and median $1.5(0-4)$ for sufficient patients, $p=0.04)$ and were more likely to be hospitalised with severe exacerbations. In the year following vitamin-D measurement $27.4 \%$ of deficient patients had an unscheduled hospitalisation for a severe exacerbation compared to $19.7 \%$ of insufficient patients and $7.1 \%$ of sufficient patients, $\mathrm{p}=0.02$.

The median $\mathrm{FEV}_{1} \%$ predicted in the study population was 72.1 (51.6-88.7) and the median forced vital capacity was 83.1 (68.3-98.7). In the vitamin-D deficient group, the median $\mathrm{FEV}_{1}$ was $68.0 \%$ (IQR $45.3-84.9 \%$ ), in the insufficient group the median $\mathrm{FEV}_{1}$ was $74.2 \%$ (IQR 59.1-93.8\%) with the median $\mathrm{FEV}_{1}$ in the sufficient group being 72.2\% (61.9-90.7), $\mathrm{p}=0.02$ by Kruskal-Wallis test. The difference between the deficient group and the insufficient group was also statistically significant $(p=0.007)$.

\section{Vitamin-D deficiency is associated with increased airways inflammation}

Figure 3 shows the relationship between vitamin-D status and measures of airways inflammation. There was a significant association between serum 25-OHD levels and each of the airway inflammation markers measured. Differences were statistically significant for myeloperoxidase (MPO) activity $(p=0.007)$, neutrophil elastase $(p=0.004)$, IL-8 $(p<0.001)$, TNF- $\alpha(p=0.03)$ and IL-1 $\beta(p=0.04)$ by Kruskal-Wallis test. There were significant differences between the deficient and insufficient groups for MPO activity $(p=0.02)$, elastase activity $(p=0.002)$, IL-8 $(p=0.002)$ but not for TNF- $\alpha(p=0.2)$ or IL-1 $\beta(p=0.07)$.

\section{Relationship between vitamin-D status, decline in lung function and survival}

Patients attended for 6 monthly spirometry for 3 years. FEV decline is expressed as a percentage of baseline $\mathrm{FEV}_{1}$ since it is known that baseline $\mathrm{FEV}_{1}$ significantly modifies the rate of absolute lung function decline, ${ }^{28}$ and vitamin-D deficient subjects had significantly lower $\mathrm{FEV}_{1}$ at baseline. 
Leicester Cough Questionnaire
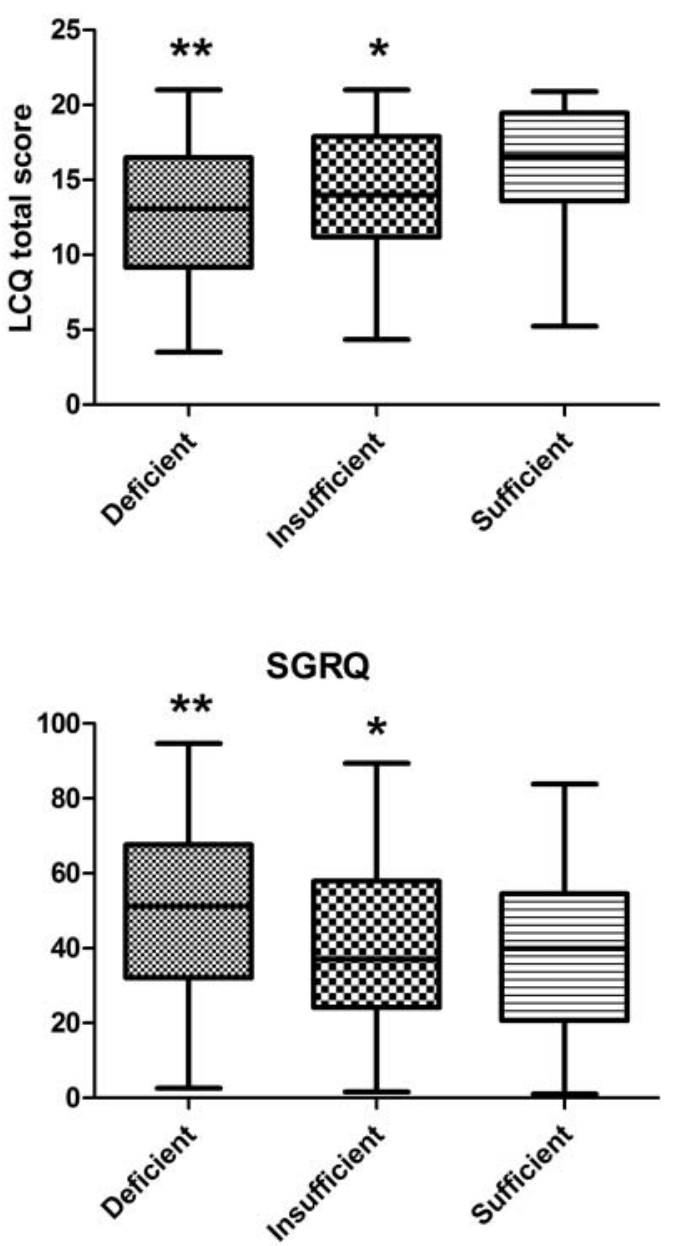

$\mathrm{FEV}_{1} \%$ predicted

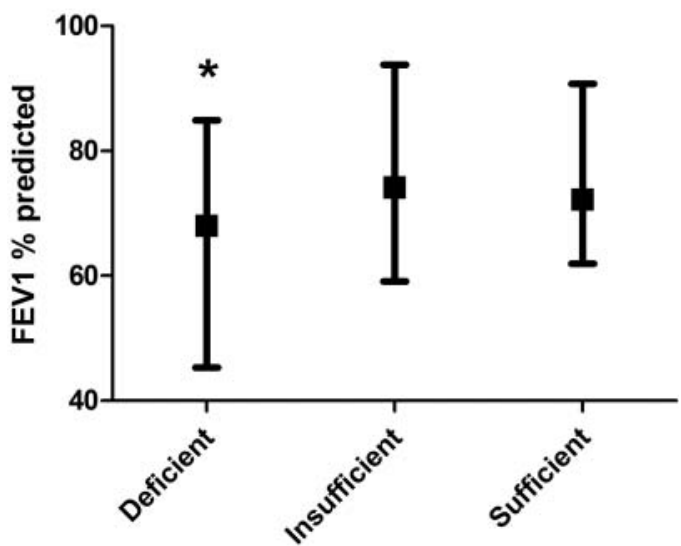

Figure 2 Relationship between vitamin-D status and the Leicester cough questionnaire (LCQ), St. Georges Respiratory questionnaire and $\mathrm{FEV}_{1} \%$ predicted. For LCQ and St. Georges Respiratory Questionnaire (SGRQ), the graph shows median and IQR (boxes) with range (bars). For $\mathrm{FEV}_{1}$, median with IQR is shown. ${ }^{* *} \mathrm{p}<0.05$ for comparison between deficient and both insufficient and sufficient groups. ${ }^{*} p<0.05$ for comparison with sufficient group. All other comparisons $p>0.05$. (Tests for trend: LCQ $p=0.004$ by Kruskal-Wallis test, SGRQ $p=0.001$ by Kruskal-Wallis test, $\mathrm{FEV}_{1} \%$ predicted $\mathrm{p}=0.02$ by Kruskal-Wallis test).
In this analysis Vitamin-D deficient subjects had a more rapid decline in lung function, $(p=0.01)$. The difference between vitamin-D insufficient and sufficient subjects was not significant (figure 4).

There were 30 deaths in this cohort over the course of 3 years (7.5\%). The cause of death was bronchiectasis related in 16 cases (listed as exacerbation of bronchiectasis, end-stage bronchiectasis, pneumonia, lower respiratory tract infection or chronic respiratory failure). Four patients died of myocardial infarction, two patients died of lung cancer, three patients died of non-pulmonary malignancy (pancreatic, colonic and nasal carcinoma respectively), one patient each died of the following causes: sepsis, stroke, severe cardiac failure, alcoholic liver disease and postoperative complications.

18 deaths occurred in the deficient cohort, 11 deaths occurred in the insufficient cohort and one death occurred in the sufficient cohort. The relationship between vitamin D status and survival was not statistically significant (log rank test $\mathrm{p}=0.4$ ) (figure 4). In Cox proportional hazard regression analysis, vitamin-D deficiency was not significantly associated with mortality, HR 1.30, 95\% CI 0.62 to 2.73 .

\section{Vitamin-D deficiency is not associated with reduced airway LL-37 concentration}

Based on previous data suggesting that antimicrobial peptide secretion was regulated by vitamin-D, we hypothesised that LL-37 would be reduced in the airways of patients with vitamin-D deficiency.

Our results showed the opposite, with higher levels of sputum LL-37 in the vitamin-D deficient group compared to the insufficient and sufficient groups $(\mathrm{p}=0.02$, Kruskal-Wallis test). There was a significant but weak correlation between sputum LL-37 and MPO concentration $(r=0.18, p=0.0003)$, neutrophil elastase $(r=0.27, p<0.001)$, IL-8 $(0.11, p=0.03)$, TNF- $\alpha$ $(r=0.22, p<0.001)$ and IL-1 $\beta(r=0.18, p=0.005)$ and higher levels of sputum LL-37 were evident in patients colonised with $P$ aeruginosa compared to patients without $P$ aeruginosa $(\mathrm{p}<0.001)$. No significant effect of $25-\mathrm{OHD}$ status on sputum LL-37 was evident among patients with chronic colonisation $(\mathrm{p}=0.4)$ and patients colonised with $P$ aeruginosa $(\mathrm{p}=0.8)$ suggesting that the higher frequency of colonisation and $P$ aeruginosa in the vitamin-D deficient group explained the observed differences in sputum LL-37.

\section{No effect of vitamin-D deficiency on neutrophil} phagocytosis in bronchiectasis patients

Neutrophils were isolated from 10 patients with bronchiectasis and vitamin-D deficiency and these were compared to 10 patients with vitamin-D insufficiency and 6 patients with sufficient 25-OHD levels.

No significant difference was observed in phagocytosis of either $P$ aeruginosa $(\mathrm{p}=0.9)$ or $E$ coli $(\mathrm{p}=0.9)$ when comparing groups according to 25 -OHD status (figure 5).

\section{Vitamin-D binding protein is elevated in the airways of} patients with severe bronchiectasis

There were statistically significant but weak correlations between sputum sol concentrations of VDBP and sputum MPO $(r=0.17$, $\mathrm{p}=0.002)$, elastase $(\mathrm{r}=0.17, \mathrm{p}=0.002), \mathrm{IL}-8(\mathrm{r}=0.12, \mathrm{p}=0.01)$ and IL-1 $\beta(r=0.27, p<0.0001)$. The relationship with TNF- $\alpha$ was not significant. Sputum sol levels of VDBP were 

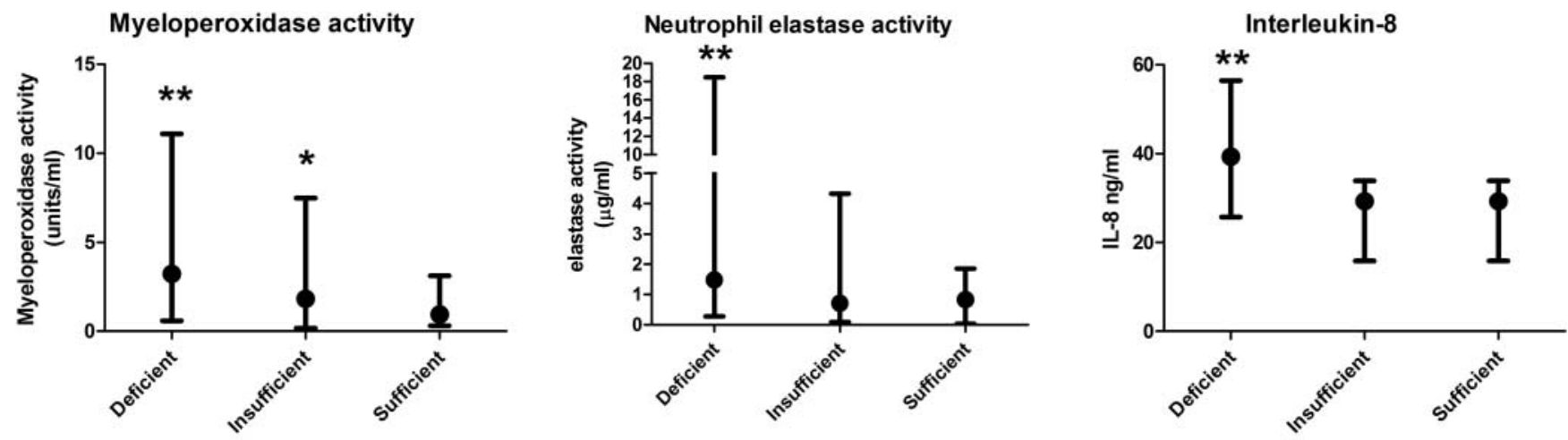

Tumour necrosis factor- $\alpha$
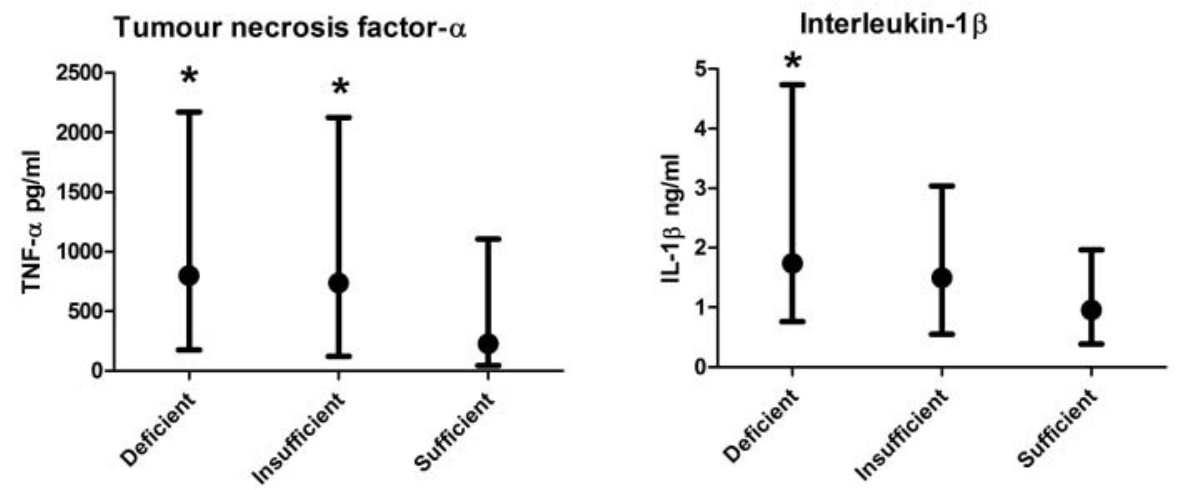

Figure 3 The relationship between vitamin-D status and markers of airway inflammation. $p<0.05$ for all comparisons by Kruskal-Wallis test. ${ }^{* *} p<0.05$ for comparison between deficient and both insufficient and sufficient groups. ${ }^{*} p<0.05$ for comparison with sufficient group. All other comparisons $\mathrm{p}>0.05$.

significantly elevated in patients with vitamin-D deficiency, compared to vitamin-D insufficiency and sufficient subjects $(\mathrm{p}<0.0001)$ (figure 6).

\section{DISCUSSION}

To our knowledge, this is the first study to investigate the role of vitamin-D in bronchiectasis. Our study has identified a strong association between vitamin-D deficiency and markers of disease severity. Compared to patients with insufficient or sufficient 25-OHD levels, patients with vitamin-D deficiency are more likely to be chronically colonised with bacteria and have evidence of increased neutrophil mediated airway inflammation. These patients suffered more frequent exacerbations and were more likely, over the course of 12 months, to be hospitalised due to a severe exacerbation. Finally, and perhaps most importantly, these patients had worse health-related quality of life and symptoms as measured using the SGRQ and LCQ.

Vitamin-D supplementation is a relatively inexpensive and safe therapy. Thus our results justify a randomised controlled trial of vitamin-D replacement therapy to determine if supplementation can improve clinical outcomes. A randomised controlled trial is essential as although this study has identified a strong association, this does not demonstrate causality. It is well recognised that vitamin-D deficiency can arise from reduced exposure to sunlight and that reduced outdoor physical activity is a common consequence of chronic lung diseases. ${ }^{8} 1416$ While we hypothesise that deficiency of vitamin-D may impair innate immunity leading to more severe bronchiectasis, it is equally
FEV $_{1}$ Decline over 3 years follow-up

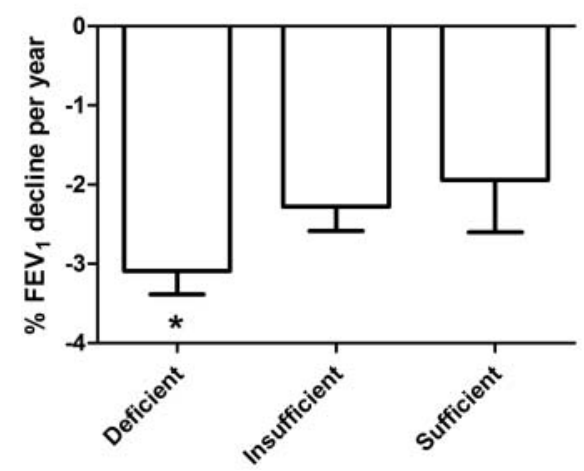

Vitamin D status and survival

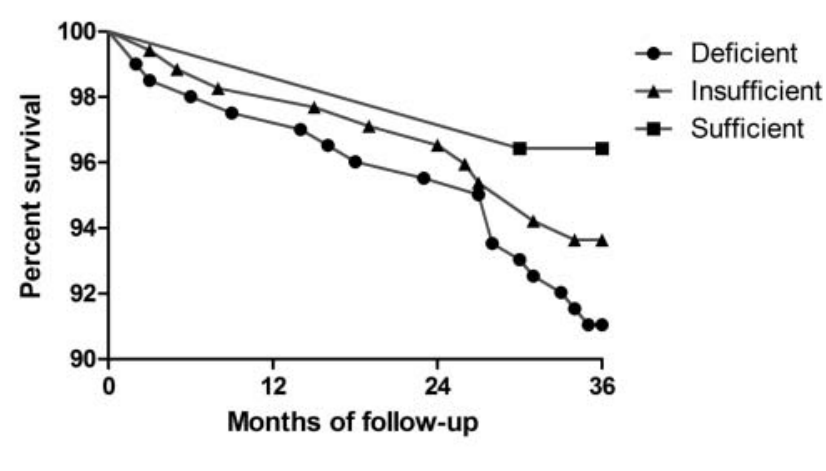

Figure 4 The relationship between vitamin-D status and lung function decline and survival over 3 years. There was a significant relationship between vitamin $D$ status and $\mathrm{FEV}_{1}$ decline, ${ }^{*} \mathrm{p}=0.01$ by Kruskal-Wallis test. The differences in survival were not statistically significant $p=0.4$. 
A

Phagocytosis of $P$. aeruginosa

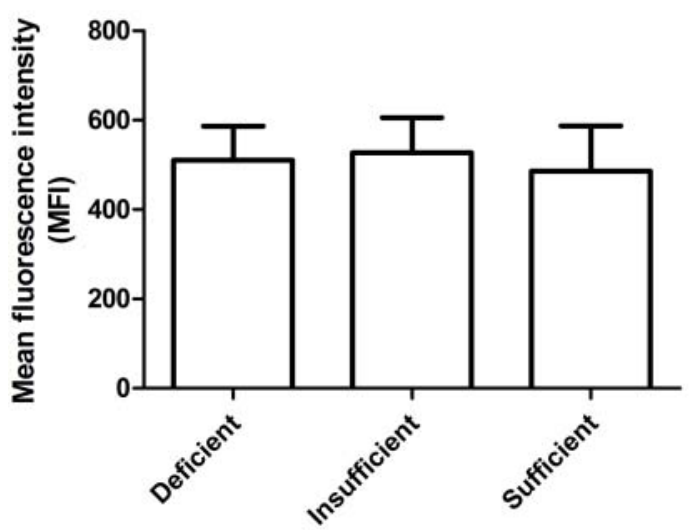

B

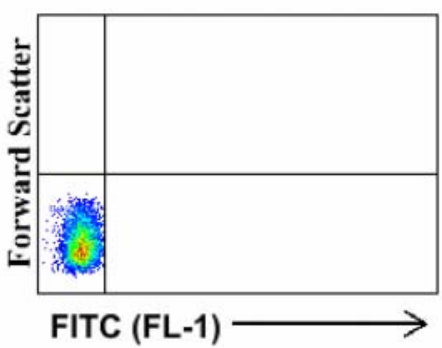

Phagocytosis of E. coli
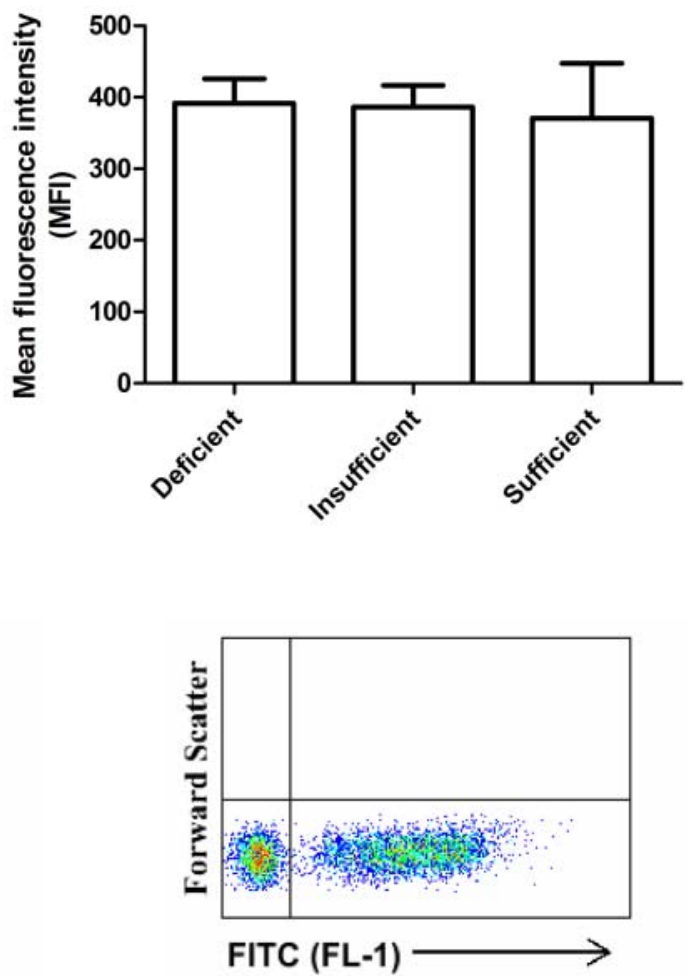

Figure 5 (A) No relationship between vitamin-D status and neutrophil phagocytosis of Pseudomonas aeruginosa or Eschericia coli. $\mathrm{N}=10$ patients with vitamin-D deficiency compared to 10 patients with vitamin-D insufficiency and six patients with sufficient 25 -hydroxyvitamin levels. $p>0.05$ for all comparisons. (B) Representative flow cytometry plots are shown: the left panel shows neutrophils not exposed to bacteria. The right panel shows neutrophils exposed to Fluorescein isothiocyanate (FITC) labelled PA01 for $30 \mathrm{~min}$.

possible that more severe bronchiectasis leads to vitamin-D deficiency through reduced outdoor physical activity.

It is for this reason that we sought to investigate the potential mechanism underlying the association between vitamin-D deficiency and airway inflammation in bronchiectasis. It has previously been shown that the powerful antimicrobial peptide

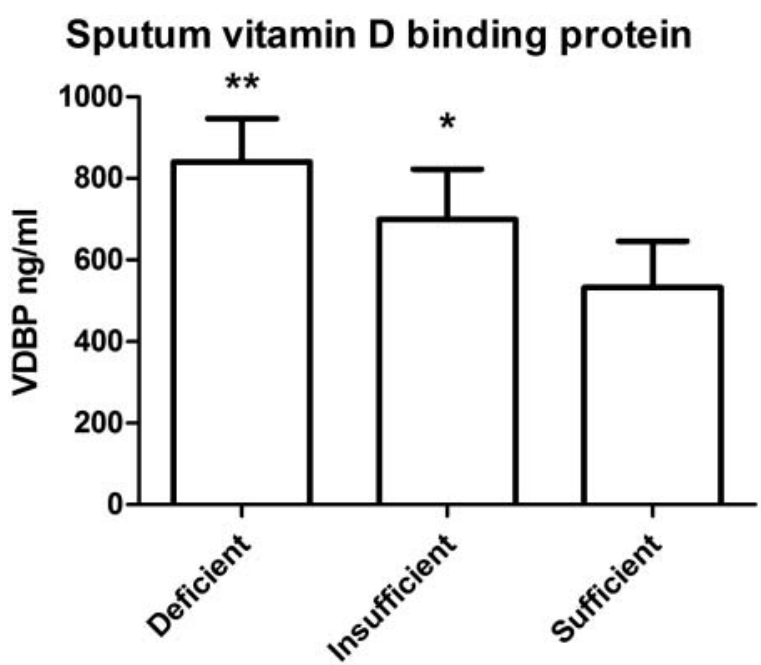

Figure 6 Vitamin-D deficient patients have higher levels of vitamin-D binding protein in sputum sol, $\mathrm{p}<0.0001$ by Kruskal-Wallis test.

${ }^{* *} p<0.05$ for comparison between deficient and both insufficient and sufficient groups. ${ }^{*} p<0.05$ for comparison with sufficient group.
LL-37 is regulated by Vitamin-D. LL-37 has activity against $P$ aeruginosa ${ }^{10}$ and therefore we hypothesised that the excess of bacterial colonisation and particularly $P$ aeruginosa in vitamin-D deficient subjects may be due to reduced production of LL-37. To the contrary, however, this study found a higher level of LL-37 in the airways of vitamin-D deficient subjects. This suggests that while vitamin-D may regulate LL-37 production in vitro, this is of little consequence in vivo, at least in the context of the bronchiectasis lung. Since bronchiectasis is a neutrophil driven disorder, ${ }^{1}$ we hypothesised that patients with vitamin-D deficiency may have a failure of neutrophil phagocytosis and killing of micro-organisms. We observed, however, no difference in neutrophil phagocytosis of $E$ coli or $P$ aeruginosa between vitamin-D deficient patients, vitamin-D sufficient patients or controls subjects.

We therefore focussed our investigation on the role of VDBP. VDBP is widely expressed by inflammatory cells and has important effects on neutrophils and macrophages, the primary inflammatory cells present in the bronchiectasis lung. ${ }^{15}$ VDBP promotes neutrophil recruitment by augmenting the chemotactic effects of complement component $\mathrm{c} 5 \mathrm{a}$ and also contributes to macrophage activation. ${ }^{17}{ }^{29}$ We found that higher levels of VDBP in sputum correlated with higher levels of neutrophil MPO and elastase and increased concentrations of IL-8 and IL-1 $\beta$. The relationship between VDBP in the airway and neutrophil mediated inflammation provides a potential mechanism for the link between vitamin-D and disease severity. Shah et $a l^{30}$ demonstrated that treatment with vitamin-D prevented C5a/VDBP co-chemotactic activity, suggesting a mechanism whereby treatment with vitamin-D could reduce neutrophil 
recruitment. Our results extend the observations of Wood et $a l^{17}$ who showed, in populations of patients with $\alpha-1$-antitrypsin deficiency and chronic obstructive pulmonary disease (COPD), an association between high levels of VDBP and lung function impairment. The authors also demonstrated the importance of macrophage activation by VDBP in vitro and therefore identified a potential mechanism for the harmful effects of VDBP. ${ }^{17}$ Our work extends these observations to patients with bronchiectasis and also links elevated levels of VDBP with a number of other important clinical indices including exacerbation frequency and airways inflammation. The level of VDBP is linked to circulating vitamin-D through alterations in vitamin-D bioavailability but also through single nucleotide polymorphisms in the VDBP gene that lead to both increased VDBP and also reduce serum vitamin-D. ${ }^{15-17}$ Janssens et $a l^{16}$ showed a clear relationship between the rs7041 polymorphism in the VDBP gene and COPD. This allele causes reduced serum levels of 25-OHD and also increases sputum VDBP. ${ }^{16}$

This study demonstrated a greater percentage $\mathrm{FEV}_{1}$ decline in the vitamin-D deficient group compared to the other two groups over a 3 year period. It is known that baseline $\mathrm{FEV}_{1}$ significantly modifies the rate of $\mathrm{FEV}_{1}$ decline and therefore we analysed this data as a percentage decline from baseline $\mathrm{FEV}_{1}{ }^{28}$ This data suggests a possible association between Vitamin-D deficiency and progression of disease, although as these patients also had a higher frequency of chronic colonisation, more exacerbations and more severe exacerbations, a causal association cannot be clearly established.

There is a reported association between vitamin-D deficiency and multiple respiratory disorders such as asthma, chronic obstructive pulmonary disease, respiratory infections and tuberculosis. $^{31-33}$ This suggests that either vitamin-D is essential for normal immune function in the airway, or that all of these studies are affected by similar confounders. Randomised controlled trials are crucial to determine if vitamin-D is a viable therapy for bronchiectasis or other respiratory disorders. The first such trial of vitamin-D replacement in COPD has now been reported. Lehouck $e a^{31}$ randomised 182 patients with moderate to very severe COPD and a history of recurrent exacerbations to high dose vitamin-D supplementation or placebo. There was no effect after 12 months on exacerbation frequency overall, but a subgroup of patients with severe vitamin-D deficiency $(<10 \mathrm{ng} / \mathrm{ml}$ at baseline) had a significant reduction in exacerbations (rate ratio $0.5795 \%$ CI 0.33 to $0.98, \mathrm{p}=0.04$ ). We believe the present study justifies a trial of vitamin-D replacement in bronchiectasis, a disease with few effective treatments.

Our study has some limitations. First, it is well recognised that different vitamin-D assays may give different results. ${ }^{34} \mathrm{We}$ used an immunoassay method that correlates well with other methods such as radioimmunoassay and investigators should determine the appropriate cut-off to define vitamin-D deficiency using their own assay and in their own population. The definition of vitamin-D deficiency is not universally accepted and so we have presented data using two vitamin-D cut-offs, both demonstrating a significant association between vitamin-D deficiency and disease severity. Vitamin-D and calcium supplementation is available without prescription and therefore, although full medication histories including 'over the counter' medication use were taken at study entry, we cannot exclude the possibility that some patients may have received vitamin-D or calcium supplementation during the study.

\section{CONCLUSION}

Vitamin-D deficiency is common in bronchiectasis and is associated with disease severity. High levels of VDBP in sputum are associated with airway inflammation and disease severity. Future studies to explore the therapeutic potential of modifying the vitamin-D axis in bronchiectasis are justified.

Contributors All authors participated in study design, data analysis and interpretation of the data. All authors were involved in writing and revising the article prior to submission.

Funding This study was funded by Chest Heart and Stroke Scotland.

\section{Competing interests None.}

Ethical approval The study was approved by the South East Scotland Research Ethics Committee and all participants gave written informed consent to participate.

Provenance and peer review Not commissioned; externally peer reviewed.

\section{REFERENCES}

1 Murray MP, Hill AT. Non-cystic fibrosis bronchiectasis. Clin Med 2009;9:164-9.

2 Pasteur MC, Bilton D, Hill AT. British Thoracic Society guideline for non-CF bronchiectasis. Thorax 2010;65(suppl 1):i1-58.

3 Pasteur MC, Helliwell SM, Houghton SJ, et al. An investigation into causative factors in patients with bronchiectasis. Am J Respir Crit Care Med 2000; 162(4 pt 1):1277-84.

4 Martinez-Garcia MA, Soler-Cataluna JJ, Perpina-Tordera M, et al. Factors associated with lung function decline in adults patients with stable non-cystic fibrosis bronchiectasis. Chest 2007;132:1565-72.

5 Angrill J, Agusti C, De Celis R, et al. Bronchial inflammation and colonization in patients with clinically stable bronchiectasis. Am J Respir Crit Care Med 2001;164:1628-32.

6 Loebinger MR, Wells AU, Hansell DM, et al. Mortality in bronchiectasis: a long-term study assessing the factors influencing survival. Eur Respir J 2009;34:843-9.

7 Rhein HM. Vitamin-D deficiency is widespread in Scotland. BMJ 2008;336:1451.

8 Hyppoenen E, Power C. Hypovitaminosis D in British adults at age $45 \mathrm{y}$ : study of dietary and lifestyle predictors. Am J Clin Nutr 2007;85:860-8.

9 van Etten E, Mathieu C. Immunoregulation by 1,25-dihydroxyvitamin-D3: basic concepts. J Steroid Biochem Mol Biol 2005:97:93-101.

10 Wang TT, Nestel FP, Bourdeau V, et al. 1,25-dihydroxyvitamin-D3 is a direct inducer of antimicrobial peptide gene expression. J Immunol 2004;173:2909-12.

11 Barlow PG, Beaumont PE, Cosseau C, et al. The human cathelicidin LL-37 preferentially promotes apoptosis of infected airway epithelium. Am J Respir Cell Mol Biol 2010;43:692-702.

12 Janssens W, Nuytten $H$, Dupont L, et al. Genomic copy number determines functional expression of \{beta\}-defensin 2 in airway epithelial cells and associates with chronic obstructive pulmonary disease. Am J Respir Crit Care Med 2010;182:163-9.

13 Zosky GR, Berry LJ, Elliot JG, et al. Vitamin-d deficiency causes deficits in lung function and alters lung structure. Am J Respir Crit Care Med 2011;183:1336-43.

14 Holick MF. Vitamin-d deficiency. N Engl J Med 2007;357:266-81.

15 Chishimba L, Thickett DR, Stockley RA, et al. The vitamin-D axis in the lung: a key role for vitamin-D-binding protein. Thorax 2010;65:456-62.

16 Janssens W, Bouillion $\mathrm{R}$, Claes $\mathrm{B}$, et al. Vitamin-d deficiency is highly prevalent in COPD and correlates with variants in the vitamin-d-binding gene. Thorax 2010;65:215-20.

17 Wood AM, Bassford C, Webster $\mathrm{P}$, et al. Vitamin-d binding protein contributes to COPD by activation of alveolar macrophages. Thorax 2011;66:205-10.

18 Hypponen $E$, Turner $S$, Cumberland $P$, et al. Serum 25-Hydroxyvitamin D measurement in a large population survey with statistical harmonization of assay variation to an international standard. J Clin Endocrinol Metab 2007:92:4615-22.

19 Carter GD. 25-Hydroxyvitamin D assays: the quest for accuracy. Clin Chem 2009;55:1300-2.

20 White AJ, Gompertz S, Bayley DL, et al. Resolution of bronchial inflammation is related to bacterial eradication following treatment of exacerbations of chronic bronchitis. Thorax 2003:58:680-5.

21 Murray MP, Govan JR, Doherty CJ, et al. A randomized controlled trial of nebulized gentamicin in non-cystic fibrosis bronchiectasis. Am J Respir Crit Care Med 2010;183(4):491-9.

22 Miller MR, Hankinson J, Brusasco V, et al. Standardisation of spirometry. Eur Respir J 2005;26:319-38.

23 Wilson CB, Jones PW, O'Leary CJ, et al. Validation of the St. Georges respiratory questionnaire in bronchiectasis. Am J Respir Crit Care Med 1997;156(2 pt 1):536-41. 
24 Murray MP, Turnbull K, MacQuarrie S, et al. Validation of the Leicester cough questionnaire in non-cystic fibrosis bronchiectasis. Eur Respir J 2009;34:125-31.

25 Stockley RA, Bayley DL. Validation of assays for inflammatory mediators in sputum. Eur Respir J 2000;15:778-81.

26 Haslett C, Guthrie LA, Kopaniak MM, et al. Modulation of multiple neutrophil functions by preparative methods or trace concentrations of bacterial lipopolysaccharide. Am J Pathol 1985;119:101-10.

27 Farnworth SL, Henderson NC, Mackinnon AC, et al. Galectin-3 reduces the severity of pneumococcal pneumonia by augmenting neutrophil function. Am J Pathol 2008:172:395-405.

28 Vestbo J, Edwards LD, Scanlon PD, et al. Changes in forced expiratory volume in 1 second over time in COPD. N Engl J Med 2011:365:1184-92.

29 Kew RR, Webster RO. Gc-globulin (vitamin-D binding protein) enhances the neutrophil chemotactic activity of $\mathrm{C} 5 \mathrm{a}$ and $\mathrm{C} 5 \mathrm{a}$ des Arg. J Clin Invest 1988;82:364-9.
30 Shah AB, DiMartino SJ, Trujillo G, et al. Selective inhibition of the C5a chemotactic cofactor function of the vitamin-d binding protein by $1,25(\mathrm{OH}) 2$ vitamin-d3. $\mathrm{Mol}$ Immunol 2006:43:1109-15.

31 Lehouck A, Mathieu C, Carremans C, et al. High doses of vitamin-d to reduce exacerbations in chronic obstructive pulmonary disease: a randomized trial. Ann Intern Med 2012;156:105-14.

32 Wejse C, Gomes VF, Rabna P, et al. Vitamin-d as supplementary treatment for tuberculosis: a double-blind, randomized, placebo controlled trial. Am J Respir Crit Care Med 2009;179:843-50.

33 Martineau AR, Timms PM, Bothamley GH, et al. High-dose vitamin-d(3) during intensive-phase antimicrobial treatment of pulmonary tuberculosis: a double-blind randomised controlled trial. Lancet 2011:377:242-50.

34 Barake M, Daher RT, Salti I, et al. 25-Hydroxyvitamin D assay variations and impact on clinical decision making. J Clin Endocrinol Metab 2012:97: 835-43. 